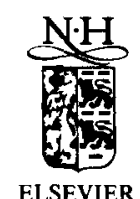

ELSEVIER

Journal of Geometry and Physics 18 (1996) 1-24

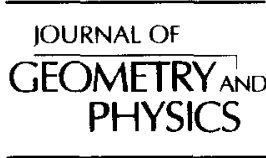

\title{
Complexification of gauge theories
}

\author{
R. Loll ${ }^{\mathrm{a}, *}$, J.M. Mourão ${ }^{\mathrm{a}, 1}$, J.N. Tavares ${ }^{\mathrm{b}, \mathrm{c}}$ \\ ${ }^{a}$ Center for Gravitational Physics and Geometry Physics Department, \\ Pennsylvania State University, University Park, PA 16802-6300, USA \\ ${ }^{b}$ Dept. Mathemática Pura, Fac. Ciências, Univ. Porto, 4000 Porto, Portugal \\ ${ }^{c}$ Dept. Física, Inst. Superior Técnico, 1096 Lisboa, Portugal
}

Received 24 May 1994

\begin{abstract}
For the case of a first-class constrained system with equivariant momentum map, we study the conditions under which the double process of reducing to the constraint surface and dividing out by the group of gauge transformations $G$ is equivalent to the single process of dividing out the initial phase space by the complexification $G_{\mathbb{C}}$ of $G$. For the particular case of a phase space action that is the lift of a configuration space action, conditions are found under which, in finite dimensions, the physical phase space of a gauge system with first-class constraints is diffeomorphic to a manifold imbedded in the physical configuration space of the complexified gauge system. Similar conditions are shown to hold for the infinite-dimensional example of Yang-Mills theories. As a physical application we discuss the adequateness of using holomorphic Wilson loop variables as (generalized) global coordinates on the physical phase space of Yang-Mills theory.
\end{abstract}

Keywords: Gauge theories; Complexification; Constrained systems; Momentum maps 1991 MSC: 81T13

\section{Introduction}

Complex gauge theories, i.e. theories with a complex group of gauge transformations, have recently appeared in several physical applications. The hamiltonian (constraint) equations of $3+1$ general relativity simplify significantly when written in terms of the $S L(2, \mathbb{C})$ Ashtekar connection and its canonically conjugate variable, the densitized triad [6]. On the

\footnotetext{
* Corresponding author.

' On leave of absence from Dept. Física, Inst. Sup. Técnicn, 1096 Lisboa, Portugal.
} 
other hand, it has been shown that general relativity with a positive cosmological constant in $2+1$ dimensions corresponds to a Chern-Simons theory with gauge group $S L(2, \mathbb{C})[27]$.

Motivated by these examples, we study the geometric properties of a wide class of complex gauge theories obtained by the "complexification" of real gauge theories. Most of our rigorous analysis takes place in finite dimensions, where the analogous setting is that of hamiltonian gauge models with first-class constraints. In the infinite-dimensional case we prove our main result for Yang-Mills theories with a compact structure group.

Our investigation is purely kinematical and concerns properties of the big and reduced phase and configuration spaces. In the case of phase spaces that are cotangent bundles and where the action of the group of gauge transformations is the lift of an action on configuration space, it is shown that under reasonable conditions the physical phase space of the real, kinematic gauge theory $\left(Q, L_{G}, Q_{\mathrm{ph}}=Q / L_{G}\right)$ is diffeomorphic (see Theorems 3 and 5) to an open submanifold $\mathcal{C}^{\text {sat }} / L_{G_{\mathfrak{C}}}$ of the physical configuration space $Q_{\mathrm{ph}}^{\mathbb{C}}$ of the complex gauge theory $\left(Q^{\mathbb{C}}, L_{G_{\mathbb{C}}}, Q_{\mathrm{ph}}^{\mathbb{C}}\right)$, which is the complexification of $\left(Q, L_{G}, Q_{\mathrm{ph}}\right)$ (see Definition 1). This result is shown to hold also in the more general case of phase spaces where the action of the group of gauge transformations $G$ possesses an equivariant momentum map.

The action of the real group $G$ on the phase space $\mathcal{P}$ is extended to an action of $G_{\mathbb{C}}$ by having the imaginary generators act appropriately in the directions orthogonal to the constraint surfaces. Note that the action of $G_{\mathbb{C}}$ will in general be symplectic only if we restrict it to the real subgroup $G$.

When the saturation $\mathcal{C}^{\text {sat }}$ (containing all points of $\mathcal{P}$ that can be reached from the constraint surface $\mathcal{C}$ by a complex gauge transformation) is dense in $Q^{\mathbb{C}}$ - which was the case in all examples studied - the above-mentioned diffeomorphism implies that in order to find the physical, reduced phase space for the system under consideration, the double process of restricting to the constraint surface $\mathcal{C} \subset \mathcal{P}$ and dividing out by the real gauge transformations is equivalent to dividing out by the complex gauge transformations. This is in accordance with the expectation that the complex gauge orbits have twice the dimension of the real gauge orbits.

An analogous result for Chern-Simons theory was proven in [15], namely, that the cotangent bundle of the physical phase space of a Chern-Simons theory with compact gauge group $K$ is diffeomorphic to a dense submanifold in the physical phase space of the theory with complex gauge group $K_{\mathrm{C}}$ [27].

The equivalence between the physical phase space $\mathcal{P}_{\text {ph }}$ of the real gauge theory and the physical configuration space $Q_{\mathrm{ph}}^{\mathbb{C}}$ of the complex gauge theory may play an important role for both theories. On the one hand, the quantization of the complex theory, by analogy with the Palatini theories [8], is expected to be facilitated by the existence of additional structures in $Q_{\mathrm{ph}}^{\mathrm{C}}$, induced from $\mathcal{P}_{\mathrm{ph}}$. It also allows us in principle to relate the Hilbert spaces associated with the quantization of the complex theory with the better-understood Hilbert spaces of the quantization of the real theory (an example is given by the generalization to a complex gauge group of the Chern-Simons theory in $2+1$ dimensions by Witten [28]). On the other hand, global (generalized) coordinates in $Q_{\mathrm{ph}}^{\mathrm{C}}$ may be used in $\mathcal{P}_{\mathrm{ph}}$. This motivates the use 
of "holomorphic Wilson loop variables" as global coordinates on the physical phase space of Yang-Mills theories with real gauge groups, and gives further justification to the use of such variables in general relativity, written in the Ashtekar variables (cf. Section 5).

We do not address here the general question of how a dynamical principle can be incorporated into our framework. Standard hamiltonians for gauge theories may not be physically meaningful in the complexified theory. For example, it is well known that the usual YangMills action proportional to $\operatorname{Tr} F F$ for a complex gauge group $G$ leads to a non-positive energy. Note also that the theories we cited at the beginning of the introduction are examples of the so-called generally covariant theories, whose particular properties render them meaningful in spite of the presence of complex structures. Furthermore, as illustrated by the example of $3+1$ gravity in terms of Ashtekar variables, it may be necessary to complement the theory by a set of reality conditions, projecting out the sector of physical states.

The present work is organized as follows. In Section 2 we describe the class of gauge theories under study. The main result relating $\mathcal{P}_{\mathrm{ph}}$ and $Q_{\mathrm{ph}}^{\mathrm{C}}$ (i.e. $\mathcal{P} / L_{G_{\mathrm{C}}}$ ) is formulated and proven in Section 3. In Section 4 we study three illustrative finite-dimensional examples, with the groups of gauge transformations $G=\mathbb{R}^{n}, G=S O(n)$, and $G=U(n)$ respectively. The latter is an example of an action that is not the lift of a configuration space action. Its physical phase space is a complex Grassmann manifold. In Section 5 we demonstrate that our techniques are applicable on the infinite-dimensional phase space of Yang-Mills theory on an arbitrary three-dimensional compact and oriented manifold. We also comment on how general relativity in terms of the Ashtekar variables can be viewed in the same framework. In Section 6 we present our conclusions.

\section{Gauge theories in the hamiltonian formalism}

In order to set the stage for the field theoretic application, we first study the geometry of the analogous finite-dimensional hamiltonian systems with symmetry which, in Dirac's terminology, are gauge models with a set of first-class constraints.

Let the finite-dimensional manifold $Q$ be the "big" configuration space of such a gauge system, and let $L_{G}$ denote a proper, but not necessarily free action of a Lie group $G$ as the group of gauge transformations on $Q$. (Recall that an action $L_{G}$ is called proper if the inverse images of compact sets under the map $(g, x) \rightarrow\left(L_{g} x, x\right)$ are again compact.) Assuming that $Q / L_{G}$ (possibly after excluding singular orbits from $Q$ ) has the structure of a differentiable manifold, this quotient space is known as the physical configuration space of the system:

$$
Q_{\mathrm{ph}}=Q / L_{G} .
$$

The resulting triplet

$$
\left(Q, L_{G}, Q_{\mathrm{ph}}\right)
$$


will be called a kinematic gauge theory. In our current investigation we will not address the question of how a physical, gauge-invariant dynamics can be introduced into this setting in a meaningful way.

The big phase space of the gauge theory (2) is the cotangent bundle $\mathcal{P}=T^{*} Q$, with the canonical symplectic form $\Omega$. The gauge transformations of the big configuration space, $L_{g}, g \in G$, lift uniquely to symplectic ( $\Omega$-preserving) gauge transformations $\tilde{L}_{g}, g \in G$, of $\mathcal{P}$. Note that our results also apply when the phase space $\mathcal{P}$ is not of the form of a cotangent bundle and/or the symplectic action of the symmetry group $G$ is not the lift of an action on the configuration space (cf. Section 4.3).

We will now introduce the notion of a momentum map $[1,14,3]$, which is a useful tool in the abstract formulation of hamiltonian systems with symmetry. The components of the momentum map are just the conserved quantities associated with that symmetry (for example, the components of the angular momentum of a particle in the presence of rotational symmetry). For gauge systems, the conserved quantities are the first-class constraints, which are required to vanish for physical configurations. The constraints define a submanifold in phase space, the so-called constraint surface $\mathcal{C}$, which can alternatively be described as the zero level set of the corresponding momentum map.

For the class of gauge systems with action $\widetilde{L}_{G}$ we are considering, the momentum map $\mu: \mathcal{P} \rightarrow \mathcal{G}^{*}\left(\mathcal{G}^{*}\right.$ denoting the dual of the Lie algebra $\mathcal{G}$ of $G$ ) always exists and is constructed as follows: for each algebra element $\xi \in \mathcal{G}$, let the vector field $\xi^{\mathcal{P}} \in \mathcal{X}(\mathcal{P})$ be the infinitesimal generator of the action $\widetilde{L}_{G}$ associated to $\xi$,

$$
\xi^{\mathcal{P}}(p)=\left.\frac{\mathrm{d}}{\mathrm{d} t}\right|_{t=0} \tilde{L}_{\exp (t \xi)} p, \quad \forall p \in \mathcal{P} .
$$

Each such $\xi^{\mathcal{P}}$ is a globally hamiltonian vector field on $\mathcal{P}$, with hamiltonian function $\mu^{\xi}$ : $\mathcal{P} \rightarrow \mathbb{R}$ given by

$$
\mu^{\xi}\left(\alpha_{q}\right)=\alpha_{q}\left(\xi^{Q}(q)\right), \quad \forall \alpha_{q}=p \in T^{*} Q
$$

where $\xi^{Q}$ is the infinitesimal generator of gauge transformations on $Q$, and we have identified a phase space point $p$ with the corresponding one-form $\alpha$ on $Q$, where $\alpha_{q} \in T_{q}^{*} Q$ with $q=\tilde{\pi}(p)$ ( $\tilde{\pi}$ denoting the projection to the base space $Q$ ). Here $\mu^{\xi}$ is of course the first-class constraint associated with the generator $\xi$ of the group of gauge transformations.

We now collect the maps $\mu^{\xi}$ into a unique momentum map $\mu: \mathcal{P} \rightarrow \mathcal{G}^{*}$ by defining, for all $p \in \mathcal{P}$ and $\xi \in \mathcal{G}$

$$
\mu(p) \bullet \xi:=\mu^{\xi}(p)
$$

where $\bullet$ denotes the duality between $\mathcal{G}$ and $\mathcal{G}^{*}$. The momentum map (4), (5) is $\mathrm{Ad}^{*}$ equivariant [1], i.e.

$$
\operatorname{Ad}_{g^{-1}}^{*} \circ \mu=\mu \circ \tilde{L}_{g}, \quad \forall g \in G
$$

This statement implies that the first-class constraints form a true Lie algebra with respect to the Poisson brackets on $\mathcal{P}$. In more general cases of phase spaces and symmetry group actions we will assume that the $G$-action has an $\mathrm{Ad}^{*}$-equivariant momentum map. 
In order to obtain the physical phase space of the kinematic gauge theory $\left(Q, L_{G}, Q_{\mathrm{ph}}\right)$, we must use the momentum map twice. We first have to restrict the phase space $\mathcal{P}$ to the constraint surface $\mathcal{C}$, defined as the zero level set of the momentum map

$$
\mathcal{C}=\{p \in \mathcal{P}: \mu(p)=0\} .
$$

Furthermore, by equivariance the components $\mu^{\xi}$ of the momentum map generate gauge transformations $\widetilde{L}_{g}$ on $\mathcal{C}$, hence to obtain the physical phase space $\mathcal{P}_{\mathrm{ph}}$ we have to perform the quotient

$$
\mathcal{P}_{\mathrm{ph}}=\mathcal{C} / \tilde{L}_{G}
$$

In the present paper we show that in some physically relevant cases the double process in phase space of constraining to $\mathcal{C}$ and quotienting out by the real gauge group action of $G$ is equivalent to the one-stage process of quotienting out by an appropriate action of the complex group $G_{\mathbb{C}}$.

Recall that for the setting described above and for the case that the group $G$ acts freely on $\mathcal{P}$, the following reduction theorem holds (see, for example, [19] and references therein; the present formulation is taken from [1], Theorem 4.3.1).

Theorem 1. Let $(\mathcal{P}, \Omega)$ be a symplectic manifold on which the Lie group $G$ acts symplectically and let $\mu: \mathcal{P} \rightarrow \mathcal{G}^{*}$ be an $\mathrm{Ad}^{*}$-equivariant momentum map for this action. Assume $x \in \mathcal{G}^{*}$ is a regular value of $\mu$ (i.e. for every $p \in \mu^{-1}(x), \mathrm{d} \mu_{p}$ is surjective), and that the isotropy group $G_{x}$ under the $\mathrm{Ad}^{*}$-action on $\mathcal{G}^{*}$ acts freely and properly on $\mu^{-1}(x)$. Then $\mathcal{P}_{x}=\mu^{-1}(x) / \widetilde{L}_{G_{x}}$ has a unique symplectic form $\omega_{x}$ with the property $\pi_{x}^{*} \omega_{x}=i_{x}^{*} \Omega$, where $\pi_{x}: \mu^{-1}(x) \rightarrow \mathcal{P}_{x}$ is the canonical projection and $i_{x}: \mu^{-1}(x) \rightarrow \mathcal{P}$ is the inclusion.

There is an analogous result if $x \in \mathcal{G}^{*}$ is only a weakly regular value of $\mu$ (i.e. $\mu^{-1}(x)$ is a submanifold with $\left.T_{p} \mu^{-1}(x)=\operatorname{Ker} \mathrm{d} \mu_{p}\right)$, the group $G_{x}$ therefore does not act freely, the $G_{x}$-orbits in $\mu^{-1}(x)$ are all of the same type, and hence the dimension of the isotropy group $G_{p}$ constant for all points $p \in \mu^{-1}(x)$. Moreover, if the $G$-action on $Q$ is proper and free, one can prove that $[13]$

$$
\mathcal{P}_{\mathrm{ph}}=\mathcal{C} / \widetilde{L}_{G} \cong T^{*}\left(Q / L_{G}\right) \text {, }
$$

i.e. the reduced physical phase space $\mathcal{P}_{\mathrm{ph}}=\mathcal{C} / G$, given by Theorem 1, is symplectomorphic to the cotangent space $T^{*} Q_{\mathrm{ph}}$ of the reduced physical configuration space $Q_{\mathrm{ph}}=Q / L_{G}$. Under appropriate regularity conditions, an analogous result holds for the case that the action is non-free [22], the standard action of $S O(n)$ on $\mathbb{R}^{n}$ being the prototypical example (cf. Section 4.2).

\section{Complexification of gauge theories}

A complex Lie group $H$ is a Lie group which at the same time is a complex manifold [16]. The two structures are related by demanding that the map 


$$
H \times H \rightarrow H, \quad\left(h_{1}, h_{2}\right) \mapsto h_{1} h_{2}^{-1}
$$

be holomorphic.

So far we have considered the general case of triplets $\left(Q, L_{G}, Q_{\mathrm{ph}}\right)$, without specifying whether the group $G$ is real or complex. We will call such a gauge theory complex if the group $G$ of gauge transformations is a complex Lie group. It is convenient to introduce the concept of the "complexification" of a kinematic gauge theory.

Definition 1. The kinematic gauge theory $\left(Q^{\mathbb{C}}, L_{G_{\mathbb{C}}}, Q_{\mathrm{ph}}^{\mathbb{C}}\right)$ is called a complexification of the theory $\left(Q, L_{G}, Q_{\mathrm{ph}}\right)$ iff it satisfies the following three conditions:

(i) The complex configuration space is diffeomorphic to the real phase space,

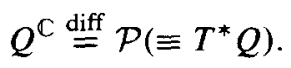

(ii) The complex gauge group can be uniquely written in the form

$$
G_{\mathbb{C}}=G \mathrm{e}^{\mathrm{i} \mathcal{G}}
$$

In case the group $G$ is compact, $G_{\mathbb{C}}$ is called the universal complexification of $G$, and $G$ is its maximal compact subgroup [16].

(iii) Using the diffeomorphism (10), the restriction of the complex action of $G_{\mathbb{C}}$ to its real subgroup $G$ coincides with the lift of the $L_{G}$-action,

$$
L_{G_{\complement} \downarrow G}=\tilde{L}_{G}
$$

This definition is motivated by the fact that the configuration space of a Yang-Mills theory with complexified structure group $K_{\mathbb{C}}$ satisfies (10)-(12), and hence can be regarded as the complexification of a Yang-Mills theory with structure group $K$ (cf. Section 5). In the present paper we study the conditions under which Eq. (10) holds at the level of the reduced spaces (if a complexification of $\left(Q, L_{G}, Q_{\mathrm{ph}}\right)$ exists), i.e. when we have

$$
Q_{\mathrm{ph}}^{\mathrm{C}} \stackrel{\text { diff }}{=} \mathcal{P}_{\mathrm{ph}},
$$

where $\mathcal{P}_{\text {ph }}$ is defined by (8).

Consider now the (not necessarily unique) complexification $\left(Q^{\complement}, L_{G_{\mathbb{C}}}, Q_{\mathrm{ph}}^{\mathbb{C}}\right.$ ) of the kinematic gauge theory $\left(Q, L_{G}, Q_{\mathrm{ph}}\right)$. The action of $G_{\mathbb{C}}$ on $\mathcal{P}$ defines a homomorphism $\tau$ of Lie algebras from the complexified Lie algebra $\mathcal{G}_{\mathbb{C}}$ into the vector fields on $\mathcal{P}$,

$$
\tau: \mathcal{G}_{\mathbb{C}}=\mathcal{G}+\mathrm{i} \mathcal{G} \longrightarrow \mathcal{X}(\mathcal{P}), \quad \tau(\xi+\mathrm{i} \eta)=\xi^{\mathcal{P}}+(\mathrm{i} \eta)^{\mathcal{P}}, \quad \xi, \eta \in \mathcal{G} .
$$

We will assume that $\mathcal{P}$ and $L_{G_{\mathbb{C}}}$ are such that the following conditions hold.

\section{Conditions 1.}

- There exists a symplectic almost-complex structure $\mathbf{J}$ on $\mathcal{P}$ such that

$$
(\mathrm{i} \eta)^{\mathcal{P}}=\mathbf{J} \eta^{\mathcal{P}}, \quad \forall \eta \in \mathcal{G}
$$


- The non-degenerate symmetric tensor $\gamma$ on $\mathcal{P}$ defined by $\Omega$ and $\mathbf{J}$ through

$$
\gamma(X, Y):=\Omega(X, \mathbf{J} Y)
$$

is a Riemannian metric, i.e. $\mathcal{P}$ is a quasi-Kähler manifold.

There are a number of conclusions that can be drawn from the existence on $\mathcal{P}$ of an almost-complex structure $\mathbf{J}$ and a Riemannian metric $\gamma$ that intertwine with the symplectic structure according to (16):

- For all linear subspaces $S \subset T_{p} \mathcal{P}$ we have

$$
\mathbf{J}\left(S^{\perp}\right)=S^{\circ},
$$

where $S^{\perp}$ denotes the subspace ( $\gamma$-)orthogonal and $S^{\curvearrowleft}$ the subspace polar symplectic (or $\Omega$-orthogonal) to $S$.

- Denoting by $\nabla \mu^{\xi}$ the gradient vector field with respect to $\gamma$ of the constraint function $\mu^{\xi}: \mathcal{P} \rightarrow \mathbb{R}$,

$$
\mathrm{d} \mu^{\xi}(Y)=\gamma\left(\nabla \mu^{\xi}, Y\right), \quad \forall Y \in \mathcal{X}(\mathcal{P}),
$$

we have

$$
\nabla \mu^{\xi}=\mathbf{J} \xi^{\mathcal{P}},
$$

i.e. the vector space perpendicular to a surface $\mu=$ const. is obtained by applying the complex structure $\mathbf{J}$ to the infinitesimal gauge generators $\xi^{\mathcal{P}}$. In fact, using (18) and the fundamental relation (16), one derives for all $Y \in \mathcal{X}(\mathcal{P})$

$$
\gamma\left(\nabla \mu^{\xi}, Y\right)=\mathrm{d} \mu^{\xi}(Y)=\Omega\left(\xi^{\mathcal{P}}, Y\right)=\gamma\left(\mathbf{J} \xi^{\mathcal{P}}, Y\right) .
$$

- For each point $p \in \mathcal{P}$, define a map $\nabla \mu_{p}: \mathcal{G} \rightarrow T_{p} \mathcal{P}$ by

$$
\nabla \mu_{p}(\xi)=\nabla \mu^{\xi}(p)=\mathbf{J} \xi^{\mathcal{P}}(p) .
$$

Identifying the tangent space $T_{p} \mathcal{P}$ with the cotangent space $T_{p}^{*} \mathcal{P}$ via the Riemannian metric $\gamma$, we see that the map $\nabla \mu_{p}: \mathcal{G} \rightarrow T_{p} \mathcal{P}$ is the adjoint of the map $\mathrm{d} \mu_{p}$ : $T_{p} \mathcal{P} \rightarrow \mathcal{G}^{*}$.

- It is easy to verify that

$$
T_{p}\left(\widetilde{L}_{\mathrm{G}} \cdot p\right)=\left(\operatorname{Ker} \mathrm{d} \mu_{p}\right)^{\circ}=\mathbf{J}\left(\operatorname{Im} \nabla \mu_{p}\right)
$$

and

$$
\operatorname{Ker}\left(\nabla \mu_{p}\right)=\mathcal{G}_{p},
$$

where $\widetilde{L}_{G} \cdot p$ denotes the $G$-orbit through the point $p \in \mathcal{P}$, and $\mathcal{G}_{p}$ the Lie algebra of the isotropy group $G_{p}$ at $p$.

Thus, if the pair $\left(L_{G_{C}}, \mathcal{P}\right)$ satisfies Condition 1, the imaginary generators of $G_{\mathbb{C}}$ are represented by vector fields $\gamma$-orthogonal to the surfaces $\mu=$ const. Note that these vector fields $\mathbf{J} \xi^{\mathcal{P}}$ are in general neither hamiltonian nor isometries. 
Note furthermore that although the $G$-action on $\mathcal{P}$ is proper, the $G_{\mathbb{C}}$-action need not be, which implies that the quotient $Q^{\mathbb{C}} / L_{G_{\mathbb{C}}}$ need not be Hausdorff. This of course can only occur since $G_{\mathbb{C}}$ is a non-compact group. Although one can make sense of the case when the $G$-action is proper and the quotient space $Q / L_{G}$ is an orbifold (i.e. no longer a smooth manifold) [9], we are not aware of a treatment or a physical interpretation for the non-Hausdorff case. Our work may be viewed as a prescription of how to deal with such gauge systems, by selecting a sufficiently well-behaved subspace of $Q^{C}$.

Let us now recall the Moncrief decomposition for gauge systems [20,4] which characterizes an orthogonal splitting of the tangent spaces $T_{p} \mathcal{P}$, where $p$ lies in the constraint surface $\mathcal{C}$. Taking into account that

$$
T_{p}\left(\tilde{L}_{G} \cdot p\right) \subset \operatorname{Ker} \mathrm{d} \mu_{p}
$$

for all points $p \in \mathcal{C},(17)-(23)$ can be summarized into the following theorem.

Theorem 2. At all points $p \in \mathcal{C} \subset \mathcal{P}$, the tangent space $T_{p} \mathcal{P}$ admits the following (orthogonal) Moncrief decomposition:

$$
T_{p} \mathcal{P}=\underbrace{\operatorname{Ker} \mathbf{d} \mu_{p} \cap \mathbf{J}\left(\operatorname{Ker} \mathrm{d} \mu_{p}\right)}_{(\mathbf{1})} \oplus \underbrace{\operatorname{Im} \nabla \mu_{p}}_{(\mathbf{2})} \oplus \underbrace{\mathbf{J}\left(\operatorname{Im} \nabla \mu_{p}\right)}_{(\mathbf{3})}
$$

the first summand being symplectic, and the last two isotropic.

At a given point $p \in \mathcal{C}$, the Moncrief decomposition (25) of $T_{p} \mathcal{P}$ has the following geometric interpretation.

- (1) $=\operatorname{Ker} d \mu_{p} \cap \mathbf{J}\left(\operatorname{Ker} \mathrm{d} \mu_{p}\right)$ can be naturally identified with the tangent space $T_{\pi(p)} \mathcal{P}_{\mathrm{ph}}$, and represents the true, physical degrees of freedom of the system.

- (2) $=\operatorname{Im} \nabla \mu_{p}$ represents infinitesimal deformations orthogonal to the constraint surface $\mathcal{C}$.

- (3) $=\mathbf{J}\left(\operatorname{Im} \nabla \mu_{p}\right)=T_{p}\left(\widetilde{L}_{G} \cdot p\right)$ is the tangent space to the gauge directions, that is, to the $G$-orbit $\widetilde{L}_{G} \cdot p$ through $p \in \mathcal{C}$.

Consider now the subset $\mathcal{C}^{\text {sat }}$ of $\mathcal{P}$ given by

$$
\mathcal{C}^{\text {sat }}:=\left\{L_{g} \cdot p: p \in \mathcal{C} \text { and } g \in G_{\mathbb{C}}\right\}
$$

We will call the set $\mathcal{C}^{\text {sat }}$ the saturation of $\mathcal{C}$ (with respect to the action of $G_{\mathbb{C}}$ ). It consists of all points that can be reached from the constraint surface by a complex gauge transformation. We prove below that $\mathcal{C}^{\text {sat }}$ is open in $\mathcal{P}$ and conjecture that it is actually dense in $\mathcal{P}$. In Section 4 we corroborate this conjecture by some examples.

The Moncrief decomposition (25) implies the "local" (in a neighbourhood of $\mathcal{C}$ ) equivalence between $\mathcal{C}^{\text {sat }} / L_{G_{\mathbb{C}}}$ and $\mathcal{P}_{\mathrm{ph}}=\mathcal{C} / \widetilde{L}_{G}$. The following propositions prove their global equivalence.

Proposition 1. The saturation $\mathcal{C}^{\text {sat }}$ is open in the phase space $\mathcal{P}$. 
Proof. By virtue of the Moncrief decomposition, $\left\{\xi_{p}^{\mathcal{P}}: \xi \in \mathrm{i} \mathcal{G}\right\}=\operatorname{Im} \nabla \mu_{p}$ is a complementary subspace to $T_{p} \mathcal{C}$ in $T_{p} \mathcal{P}$.

Therefore $\mathcal{C}^{\text {sat }}$ contains an open neighbourhood $\mathcal{U}$ of $\mathcal{C}$ in $\mathcal{P}$. Moreover, since $\mathcal{C}^{\text {sat }}$ is given by the union $\mathcal{C}^{\mathrm{sat}}=\bigcup_{g \in G_{\mathbb{C}}}\left\{L_{g} \cdot \mathcal{U}\right\}$, it follows that the saturation $\mathcal{C}^{\text {sat }}$ is open in $\mathcal{P}$.

Now, since $\mathcal{C} \subset \mathcal{C}^{\mathrm{sat}}$ and since each "real" orbit $\tilde{L}_{G} \cdot p, p \in \mathcal{C}$ is contained in a "complex" orbit $L_{G_{C}} \cdot p$, we have a map

$$
\mathcal{P}_{\mathrm{ph}}=\mathcal{C} / \tilde{L}_{G} \rightarrow \mathcal{C}^{\text {sat }} / L_{G_{\mathbb{C}}}
$$

Since our aim is to prove the equivalence between the physical phase space and the complex quotient on the right-hand side, we must show that the map (27) is a bijection. Equivalently, we must prove that each "complex" orbit $L_{G_{\mathbb{C}}} \cdot p, p \in \mathcal{C}$, contains only one "real" orbit $L_{G} \cdot p$, i.e. that the following proposition is true.

\section{Proposition 2.}

$$
\left(L_{G_{\mathbb{L}}} \cdot p\right) \cap \mathcal{C}=L_{G} \cdot p, \quad \forall p \in \mathcal{C} .
$$

Proof. It suffices to prove that

$$
\left(L_{G_{\mathbb{C}}} \cdot p\right) \cap \mathcal{C} \subset L_{G} \cdot p .
$$

Let $L_{g} \cdot p \in\left(L_{G_{\mathbb{C}}} \cdot p\right) \cap \mathcal{C}$, with $g \in G_{\mathbb{C}}$. We must show that $L_{g} \cdot p=L_{h} \cdot p$, for some real group element $h \in G$. However, $\operatorname{since} \mathcal{C}$ is a $G$-invariant submanifold of $\mathcal{P}$ and $G_{C}=G \cdot \exp (i \mathcal{G})$ by assumption, we can without loss of generality take $g$ to be of the form $g=\exp (\mathrm{i} \xi)$, with $\xi \in \mathcal{G}$.

Hence assume that

$$
L_{\exp (\mathrm{i} \xi)} \cdot p=L_{\mathrm{e}}{ }^{\mathrm{i} \xi} \cdot p \in \mathcal{C} .
$$

Let us set $q_{l}:=L_{\mathrm{e}^{\mathrm{i} \xi \xi}} \cdot p$, and consider the function $f:[0,1] \rightarrow \mathbb{R}$, defined by (see Eq. (5))

$$
f(t)=\mu^{\xi}\left(q_{t}\right) .
$$

Then, $f(0)=\mu^{\xi}(p)=0$, since $p \in \mathcal{C}=\mu^{-1}(0)$, and also $f(1)=\mu^{\xi}\left(q_{1}\right)=0$, by assumption (30). On the other hand, using (14) and (16) we compute

$$
f^{\prime}(t)=\mathrm{d} \mu^{\xi}\left(q_{t}\right)\left(\mathbf{J} \xi^{\mathcal{P}}\left(q_{t}\right)\right)=\Omega_{q_{t}}\left(\xi^{\mathcal{P}}\left(q_{t}\right), \mathbf{J} \xi^{\mathcal{P}}\left(q_{t}\right)\right)=\gamma\left(\xi^{\mathcal{P}}\left(q_{t}\right), \xi^{\mathcal{P}}\left(q_{t}\right)\right) \geq 0 .
$$

Therefore, the function $f$ must vanish on the entire interval $[0,1]$, and $q_{t} \in \mathcal{C}$, for all $t \in[0,1]$. Moreover we have, for all $t \in[0,1], \xi^{\mathcal{P}}\left(q_{t}\right)=0$ which implies $q_{t}=p$ and, in particular,

$$
L_{\mathrm{e} \dot{\xi}} \cdot p=p \in L_{G} \cdot p
$$


In conclusion, the map (27) is a bijection. Moreover, using the Moncrief decomposition and the inverse mapping theorem, one can easily prove that this map is in fact a ( $\mathrm{J}$-dependent) diffeomorphism. We have therefore proved our main result.

Theorem 3. Let $\widetilde{L}_{G}$ be a proper symplectic action of the Lie group $G$ on the symplectic manifold $P$, with an $\mathrm{Ad}^{*}$-equivariant momentum map $\mu$, and let $L_{G_{\mathbb{C}}}$ be a complexification of this action satisfying (11), (12) and Condition 1 . If 0 is a weakly regular value of $\mu$, the map (27) is a diffeomorphism between $\mathcal{C} / \widetilde{L}_{G}$ and $\mathcal{C}^{\text {sat }} / L_{G_{C}}$, where $\mathcal{C}^{\text {sat }}$ is given by (26).

Note that there may exist complex orbits in $Q^{C}$ which do not contain any real orbit $\tilde{L}_{G} \cdot p, p \in \mathcal{C}$, i.e. not every complex orbit necessarily intersects the constraint surface (see for example Sections 4.2 and 4.3). However, in the finite-dimensional examples we considered, $\mathcal{C}^{\text {sat }}$ was always dense in $\mathcal{P}$. We conjecture that this is the case for a wide class of systems and in particular for the Yang-Mills theory discussed in Section 5.

Given the diffeomorphism between $\mathcal{P}_{\mathrm{ph}}$ and $\mathcal{C}^{\text {sat }} / L_{G_{\mathbb{C}}}$, we can now pull back to $\mathcal{C}^{\text {sat }} / L_{G_{C}}$ the unique symplectic form on the physical phase space obtained from the MarsdenWeinstein reduction.

Conversely, we are interested in the question under what conditions a given kinematic gauge theory $\left(Q, L_{G}, Q_{\mathrm{ph}}\right)$ can be complexified. One necessary condition is that the vector fields $\mathbf{J} \eta^{\mathcal{P}}$ (cf. (15)) be complete, so that their infinitesimal action can be exponentiated. We also need a $G$-invariant almost complex structure $\mathbf{J}$ on the phase space $\mathcal{P}$. This is not a very strong restriction, since for compact $G$ one can always define a $G$-invariant almostKähler structure on $\mathcal{P}$. The most important condition comes from demanding that the map $\tau: \mathcal{G}_{\mathbb{C}} \rightarrow \mathcal{X}(\mathcal{P})$ constructed according to

$$
\tau(\xi+\mathrm{i} \eta):=\xi^{\mathcal{P}}+\mathbf{J} \eta^{\mathcal{P}}
$$

with $\xi, \eta \in \mathcal{G}$, define a homomorphism of Lie algebras (cf. expressions (14) and (15)). It turns out that this is the case only if the restriction of the almost-complex structure $\mathbf{J}$ to the complex $G_{\mathfrak{C}}$-orbits in $\mathcal{P}$ is integrable. All these conditions are satisfied for the finitedimensional gauge systems discussed in the next section.

After finishing this work, it came to our attention that quotient constructions similar to ours feature in some mathematical work by Ness [23], Kirwan [17] and Sjamaar [26]. Their original motivation is Mumford's categorical quotient in the context of geometric invariant theory and analogous constructions for the case of Kähler manifolds, in particular, its cohomological properties. The mathematical setting seems to differ from ours in that we require only an almost-Kähler structure on phase space (cf. Condition1). It would be interesting to see whether any of their methods could be used profitably in proving our denseness conjecture, or learn more about the physical systems that are our primary motivation. The precise relation with our results therefore deserves further attention, but is beyond the scope of this paper. 


\section{Finite-dimensional examples}

\subsection{Group of gauge transformations $\mathbb{R}^{n}$}

We first consider an example with an abelian group of gauge transformations, the kinematic gauge model $\left(Q, L_{G}, Q_{\mathrm{ph}}\right)=\left(\mathbb{R}^{m}, L_{\mathbb{R}^{n}}, \mathbb{R}^{m-n}\right)$, where $n<m$, with $\mathbb{R}^{n}$ acting freely on $\mathbb{R}^{m}$. The coordinates on the configuration space $Q$ are the $x_{l}, I=1, \ldots, m$, and the $n$ algebra generators $\xi^{i}$ of the gauge group $\mathbb{R}^{n}$ are taken to act on $Q$ by the vector fields $\partial / \partial x_{i}, i=1, \ldots, n$. The corresponding first-class constraints on $\mathcal{P}=T^{*} \mathbb{R}^{m}$ are given by $p_{i}=0, i=1, \ldots, n$, with the canonically lifted action on $\mathcal{P}$,

$$
\xi^{i} \mapsto \frac{\partial}{\partial x_{i}}, \quad i=1, \ldots, n
$$

The $(2 m-n)$-dimensional constraint surface $\mathcal{C}$ is given by

$$
\mathcal{C}=\left\{(\boldsymbol{x}, \boldsymbol{p}) \in \mathbb{R}^{2 m}: p_{i}=0, i=1, \ldots, n\right\} .
$$

The physical phase space $\mathcal{P}_{\mathrm{ph}}$ is the cotangent bundle $T^{*} \mathbb{R}^{m-n}$, parametrized by the $m-n$ coordinate pairs $\left(x_{a}, p_{a}\right), a=n+1, \ldots, m$, with the induced canonical symplectic form.

We are now looking for suitable complexifications of $\left(\mathbb{R}^{m}, L_{\mathbb{R}^{n}}, \mathbb{R}^{m-n}\right)$. Consider the complex structure $\mathbf{J}$ on $\mathcal{P}$ defined by

$$
\mathbf{J} \frac{\partial}{\partial x_{I}}=\frac{\partial}{\partial p_{I}}, \quad \mathbf{J} \frac{\partial}{\partial p_{I}}=-\frac{\partial}{\partial x_{l}}, \quad I=1, \ldots, m
$$

We use this complex structure to extend the action of $\mathbb{R}^{n}$ to one of $\left(\mathbb{R}^{n}\right) \mathbb{C}=\mathbb{C}^{n}$ by representing the "imaginary" generators as

$$
\mathrm{i} \xi^{j} \mapsto \mathbf{J} \frac{\partial}{\partial x_{j}}=\frac{\partial}{\partial p_{j}}, \quad j=1, \ldots, n .
$$

If we now consider the kinematic gauge theory $\left(\mathbb{C}^{m}, L_{\mathbb{C}^{n}}, \mathbb{C}^{m-n}\right)$ with coordinates $z_{1}=$ $x_{l}+\mathrm{i} p_{I}$ on the complex configuration space $\mathbb{C}^{m}$, and with the action of the gauge group $\mathbb{C}^{n}$ defined by

$$
\tau\left(\xi^{i}+\mathrm{i} \xi^{j}\right)=\frac{\partial}{\partial x_{i}}+\mathrm{i} \frac{\partial}{\partial p_{j}}, \quad i, j=1, \ldots, n,
$$

by Definition 1 this is a complexification of the triplet $\left(\mathbb{R}^{m}, L \mathbb{R}^{n}, \mathbb{R}^{m-n}\right)$, under the diffeomorphism $z_{I} \rightarrow\left(x_{l}, p_{I}\right)$ between $\mathbb{C}^{m}$ and $T^{*} \mathbb{R}^{m}$. Moreover, the symmetric tensor $\gamma$ on $\mathcal{P}$ constructed according to (16) is a well-defined Riemannian metric,

$$
\begin{aligned}
& \gamma\left(\frac{\partial}{\partial x_{I}}, \frac{\partial}{\partial x_{J}}\right)=\Omega\left(\frac{\partial}{\partial x_{I}}, \frac{\partial}{\partial p_{J}}\right)=\delta_{I J}, \\
& \gamma\left(\frac{\partial}{\partial x_{I}}, \frac{\partial}{\partial p_{J}}\right)=\Omega\left(\frac{\partial}{\partial x_{I}},-\frac{\partial}{\partial x_{J}}\right)=0, \\
& \gamma\left(\frac{\partial}{\partial p_{I}}, \frac{\partial}{\partial p_{J}}\right)=\Omega\left(\frac{\partial}{\partial p_{I}},-\frac{\partial}{\partial x_{J}}\right)=\delta_{I J},
\end{aligned}
$$


so that Condition 1 is satisfied. The saturation $\mathcal{C}^{\text {sat }}$ of $\mathcal{C}$ in $\mathcal{P}$ under the action of $\mathbb{C}^{n},(35)$ and (38), is all of $\mathcal{P}$, and any $\mathbb{C}^{n}$-orbit is of the form

$$
\left\{(\boldsymbol{x}, \boldsymbol{p}) \in \mathbb{R}^{2 m}: x_{i}, p_{i} \text { fixed, } i=n+1, \ldots, m\right\},
$$

and therefore contains exactly one $\mathbb{R}^{n}$-orbit of the constraint surface $\mathcal{C}$, demonstrating the desired equivalence of the quotients

$$
\mathcal{C}^{\text {sat }} / \mathbb{C}^{n} \cong \mathcal{C} / \mathbb{R}^{n}
$$

Let us now slightly modify the definition of the complex structure on $\mathcal{P}$ by defining

$$
\mathbf{J}^{\prime} \frac{\partial}{\partial x_{1}}=\frac{\partial}{\partial p_{m}}, \quad \mathbf{J}^{\prime} \frac{\partial}{\partial x_{m}}=\frac{\partial}{\partial p_{1}}, \quad \mathbf{J}^{\prime} \frac{\partial}{\partial p_{1}}=-\frac{\partial}{\partial x_{m}}, \quad \mathbf{J}^{\prime} \frac{\partial}{\partial p_{m}}=-\frac{\partial}{\partial x_{1}},
$$

and all other relations unchanged from (37). Proceeding as above leads to a complex action on $\mathbb{C}^{m}$ with

$$
\tau\left(\mathrm{i} \xi^{1}\right)=\mathrm{i} \frac{\partial}{\partial p_{m}} .
$$

A $\mathbb{C}^{n}$-orbit on $\mathcal{P}$ is now of the form

$$
\left\{(\boldsymbol{x}, \boldsymbol{p}) \in \mathbb{R}^{2 m}: p_{1}, x_{m} \text { fixed; } \boldsymbol{x}_{a}, p_{a} \text { fixed, } a=n+1, \ldots, m-1\right\} .
$$

Such an orbit does not intersect $\mathcal{C}$ unless $p_{1}=0$, in which case it contains a whole oneparameter family (labelled by $p_{m}$ ) of $\mathbb{R}^{n}$-orbits in $\mathcal{C}$, which in turn are of the form

$$
\left\{(\boldsymbol{x}, \boldsymbol{p}) \in \mathbb{R}^{2 m}: p_{i}=0, i=1, \ldots, n ; x_{a}, p_{a} \text { fixed, } a=n+1, \ldots, m\right\} .
$$

However, this does not contradict our conjecture since the metric $\gamma^{\prime}$ constructed according to $(16)$ is not Riemannian; its signature is $(-,-,+, \ldots,+)$.

\subsection{Group of gauge transformations $S O(n)$}

Let us now consider a typical non-abelian kinematic gauge theory, given by [24]

$$
\left(Q, L_{G}, Q_{\mathrm{ph}}\right)=\left(\mathbb{R}^{n}, L_{S O(n)}, \mathbb{R}^{+}\right),
$$

where $\mathbb{R}^{+}=\{r \in \mathbb{R}, 0<r<\infty\}, L_{S O(n)}$ denotes the action of $S O(n)$ in the fundamental representation,

$$
x \in \mathbb{R}^{n} \mapsto L_{A} x=A x, \quad A^{\mathrm{t}} A=\mathrm{Id}, \operatorname{det} A=1,
$$

with Id denoting the $n \times n$-identity matrix. The action $L_{S O(n)}$ is non-free for $n>2$ and the physical configuration space is the one-dimensional manifold $\mathbb{R}^{+}$(after excluding the singular orbit $\{0\})$. The canonical lift of $L_{S O(n)}$ to the phase space is

$$
\widetilde{L}_{A}: \mathcal{P} \rightarrow \mathcal{P}, \quad \widetilde{L}_{A}(x, p)=(A x, A p),
$$

where $(x, p) \in \mathcal{P}=T^{*} \mathbb{R}^{n}=\mathbb{R}^{n} \times \mathbb{R}^{n}$. 
The equivariant momentum map is, in accordance with (4),

$$
\mu(x, p)\left(T_{j k}\right)=p_{i} \mathrm{~d} x_{i}\left(T_{j k}^{Q}\right)=x T_{j k} p^{\mathrm{t}}, \quad 1 \leq j<k \leq n,
$$

where

$$
T_{j k} \in \operatorname{so}(n), \quad\left(T_{j k}\right)_{j^{\prime} k^{\prime}}=\delta_{j j^{\prime}} \delta_{k k^{\prime}}-\delta_{j k^{\prime}} \delta_{k j^{\prime}}, \quad T_{j k}^{Q}=x_{j} \frac{\partial}{\partial x_{k}}-x_{k} \frac{\partial}{\partial x_{j}} .
$$

The first-class constraints associated with (50) take the form

$$
x T_{j k} p^{\mathrm{t}}=0, \quad 1 \leq j<k \leq n,
$$

or, equivalently,

$$
x \wedge p=0,
$$

where the wedge in (52) denotes the exterior product of the two vectors $x, p \in \mathbb{R}^{n}$ and we have identified $s o(n)$ with $\Lambda^{2} \mathbb{R}^{n}$. By exterior algebra arguments, (52) implies that the constraint surface $\mathcal{C}$ is given by

$$
\mathcal{C}=\left\{(x, p) \in \mathcal{P}:(x, p)=(\lambda f, \mu f), \lambda, \mu \in \mathbb{R}, f \in \mathbb{R}^{n},\|f\|^{2}=\sum_{i=1}^{n} f_{i}^{2}=1\right\}
$$

To obtain the physical phase space, we must divide $\mathcal{C}$ by the action of $\tilde{L}_{S O(n)}$. Since the action of $S O(n)$ is transitive on the unit sphere,

$$
S^{n-1}=\left\{f \in \mathbb{R}^{n},\|f\|=1\right\} \subset \mathbb{R}^{n},
$$

we have in each $\tilde{L}_{S O(n)}$-orbit a representative

$$
\left(\lambda f_{1}, \mu f_{1}\right) \in \mathcal{C},
$$

where $f_{1}=(1,0, \ldots, 0)$. There is a residual gauge transformation

$$
\left(\lambda f_{1}, \mu f_{1}\right) \mapsto\left(-\lambda f_{1},-\mu f_{1}\right),
$$

which means that the physical phase space is a cone [24]:

$$
\mathcal{P}_{\mathrm{ph}}=\mathcal{C} / \tilde{I}_{S O(n)}=\mathbb{R}^{2} / \mathbb{Z}_{2}=\left\{[(\lambda, \mu)]:(\lambda, \mu) \in \mathbb{R}^{2}\right\}
$$

where we denote the $\mathbb{Z}_{2}$-equivalence classes by $[(\lambda, \mu)]=\{(\lambda, \mu),(-\lambda,-\mu)\}$. Alternatively, if we exclude the origin of $\mathbb{R}^{2 n}$ and choose

$$
\mathcal{P}=\mathbb{R}_{*}^{2 n}=\mathbb{R}^{2 n} \backslash\{0\},
$$

$\mathcal{P}_{\mathrm{ph}}$ becomes a non-simply connected two-dimensional manifold,

$$
\mathcal{C} / \tilde{L}_{S O(n)} \equiv \mathbb{R}_{*}^{2}=\mathbb{R}^{2} \backslash\{0\} .
$$

Let us now see how this process of constraining and quotienting can be replaced by the single step of quotienting the big phase space (58) by the action of the complex group

$$
S O(n)_{\mathbb{C}}=S O(n, \mathbb{C})
$$


Consider the complex kinematic gauge theory

$$
\left(Q^{\mathbb{C}}, L_{G_{\mathbb{C}}}, Q_{\mathrm{ph}}^{\mathbb{C}}\right)=\left(\mathbb{C}^{n}, L_{S O(n, \mathbb{C})}, \mathbb{C}^{n} / L_{S O(n, \mathbb{C})}\right)
$$

where $L_{S O(n, C)}$ denotes the standard (non-symplectic and, in fact, improper) action of $S O(n, \mathbb{C})$ on $\mathbb{C}^{n}$,

$$
L_{A}: \mathbb{C}^{n} \rightarrow \mathbb{C}^{n}, \quad L_{A} z=A z, z \in \mathbb{C}^{n}
$$

where $z=x+\mathrm{i} p,(x, p) \in \mathbb{R}^{2 n}$ and $A$ is a complex orthogonal $n \times n$-matrix with unit determinant. It is clear that the complex kinematic gauge theory (61) is a complexification of the theory (47) (see Definition I). Besides, the standard complex structure on $\mathbb{C}^{n}$ satisfies Condition 1 and, as we will show, the map (27) is a bijection. Let us demonstrate that $\mathcal{C}^{\text {sat }}$ is dense in $\mathcal{P}=\mathbb{C}^{n}$, and that in order to give the set of orbits

$$
\mathcal{P} / L_{S O(n, \mathbb{C})}=\mathbb{C}^{n} / L_{S O(n, \mathbb{C})}
$$

a differentiable structure, we must exclude the origin $\{0\}$ and the orbits of $L_{S O(n, \mathbb{C})}$ which do not intersect $\mathcal{C}$, that is

$$
\mathcal{P}_{\mathrm{ph}}=\mathcal{C} / L_{S O(n)}=Q_{\mathrm{ph}}^{\mathbb{C}}=\mathcal{P} / L_{S O(n, \mathbb{C})},
$$

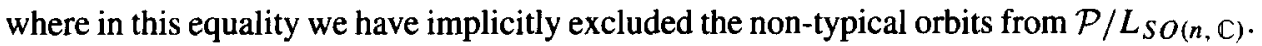
To show that $\mathcal{C}^{\text {sat }}$ is dense in $\mathcal{P}=\mathbb{C}^{n}$, we consider the following sets in $\mathbb{C}^{n}$, which are invariant under $L_{S O}(n, \mathbb{C})$,

$$
D_{w}=\left\{z \in \mathbb{C}^{m} \backslash\{0\}: z^{2}=w\right\} .
$$

It can easily be shown that $D_{0}$ does not intersect $\mathcal{C}$ and that every $D_{w}$ (for $w \neq 0$ ) contains a single orbit of $L_{S O}(m, \mathbb{C})$ and intersects $\mathcal{C}$. Then

$$
\mathcal{C}^{\text {sat }}=\bigcup_{w \in \mathbb{C}} D_{w}=\mathbb{C}^{n} \backslash D_{0}
$$

which proves that $\mathcal{C}^{\text {sat }}$ is dense in $\mathcal{P}=\mathbb{C}^{n}$ since $D_{0}$ has real codimension two. The orbits $D_{w}$ are all of the type

$$
D_{w} \equiv S O(n, \mathbb{C}) / S O(n-1, \mathbb{C})
$$

while the orbits $\{0\}$ and $D_{0}$ are not. (It can be shown that $D_{0}$ is just a single orbit, unless $n=$ 2 , when it consists of two orbits.) Therefore, in order to give $\mathcal{P} / L_{S O(n, \mathbb{C})}$ a differentiable structure, we must exclude $\{0\} \cup D_{0}$, which proves the validity of (64).

\subsection{Grassmann manifolds as the physical phase spaces of $U(m)$-gauge theories}

Let us consider an example with a symplectic action of the group $U(m)$ in $\mathcal{P}=\mathbb{R}^{2 m n}=$ $T^{*} \mathbb{R}^{m n}$, which is not the lift of an action in the configuration space $\mathbb{R}^{m n} .^{2}$ We take $\mathcal{P}=$

\footnotetext{
${ }^{2}$ We thank R. Picken for suggesting the $m=1$ example.
} 
$\mathbb{R}^{2 m n}=\mathbb{C}^{m n}$, endowed with the standard Kähler structure. The points in $\mathcal{P}$ can be considered either as complex $m \times n$-matrices,

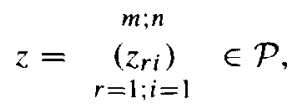

or as sets of $m$ vectors in $\mathbb{C}^{n}$ (the rows of $\left(z_{r i}\right)$ ). Let the group $U(m)$ act by left multiplication,

$$
L_{A} z=A z, \quad A \in U(m)
$$

and the group $S U(n)$ by right multiplication,

$$
L_{B} z=z B, \quad B \in S U(n) .
$$

Both actions leave the Kähler structure on $\mathcal{P}=\mathbb{C}^{m n}$-invariant.

Let us assume that the group of gauge transformations is $U(m)$. The action of $U(m)$ is free for points $z \in \mathcal{P}$ such that $\operatorname{rank}(z)=m$. Indeed, we have

$$
L_{A} z=z \Leftrightarrow \sum_{s=1}^{m}\left(A_{r s}-\delta_{r s}\right) z_{s}=0,
$$

which, if $\operatorname{rank}(z)=m$, implies that

$$
A_{r s}=\delta_{r s}
$$

The action (69) has an equivariant momentum map, given by

$$
\begin{aligned}
& \mu^{\xi_{r s}}(z)=c \delta_{r s}-\frac{1}{4}\left(z_{r} \bar{z}_{s}+\bar{z}_{r} z_{s}\right)=c \delta_{r s}-\frac{1}{2}\left(p_{r} p_{s}+x_{r} x_{s}\right), \\
& \mu^{\eta_{r s}}(z)=\frac{1}{4} \mathrm{i}\left(z_{r} \bar{z}_{s}-\bar{z}_{r} z_{s}\right)=\frac{1}{2}\left(x_{r} p_{s}-x_{s} p_{r}\right)
\end{aligned}
$$

where $u_{r} v_{s} \equiv \sum_{i=1}^{n} u_{r i} v_{s i}$, and we have set $z_{r}=x_{r}+\mathrm{i} p_{r}$. The generators $\xi_{r s}, \eta_{r s}$ form a basis in $u(m) \equiv \operatorname{Lie}(U(m))$, given by

$$
\xi_{r s}=\frac{1}{2} \mathrm{i}\left(E_{r s}+E_{s r}\right), \quad \eta_{r s}=\frac{1}{2}\left(E_{r s}-E_{s r}\right),
$$

where the $E_{r s}$ are the elementary $m \times m$-matrices

$$
\left(E_{r s}\right)_{r^{\prime} s^{\prime}}=\delta_{r r^{\prime}} \delta_{s s^{\prime}}
$$

One easily checks that the constant terms appearing on the right-hand side of (72) are the most general constants that on cohomological grounds can appear in the components of the momentum map. The first-class constraints associated with this action are

$$
\mu=0 \Leftrightarrow \mu^{\xi_{r s}}=0, \quad \mu^{\eta_{r s}}=0,
$$

or, equivalently,

$$
z_{r} \bar{z}_{s}=\delta_{r s},
$$


where we have set the constant to $c=\frac{1}{2}$. We see that the elements of the constraint surface are sets of $m$ orthonormal vectors in $\mathbb{C}^{n}$ with respect to the hermitian inner product

$$
\left\langle w, w^{\prime}\right\rangle=w \bar{w}^{\prime}=\sum_{i=1}^{n} w_{i} \bar{w}_{i}^{\prime} .
$$

The action of $U(m)$ on $\mathcal{C}$ is free as explained above. Let $z^{(0)} \in \mathcal{C}$ and $\mathcal{L}$ be the subspace of $\mathbb{C}^{n}$ spanned by $\left\{z_{r}^{(0)}\right\}_{r=1}^{m}$. Geometrically, by acting with $U(m)$ according to

$$
\left(A z^{(0)}\right)_{r}=A_{r s} z_{s}^{(0)} \text {, }
$$

we obtain all the orthonormal bases of $\mathcal{L} \subset \mathbb{C}^{n}$. The points in the physical phase space $\mathcal{P}_{\text {ph }}$ are the orbits $[z]$ of $U(m)$ in $\mathcal{C}$. There is an obvious one-to-onc map between $\mathcal{P}_{\mathrm{ph}}$ and the complex Grassmann manifold $G_{m, n}(\mathbb{C})$ of $m$-dimensional subspaces of $\mathbb{C}^{n}$,

$$
\mathcal{P}_{\mathrm{ph}} \rightarrow G_{m, n}(\mathbb{C}), \quad[z] \mapsto \mathcal{L}=\operatorname{span}\left\{z_{s}\right\}_{s=1}^{m}
$$

That this map is a diffeomorphism follows from the following proposition.

\section{Proposition 3.}

- The constraint surface $\mathcal{C}$ is an orbit in $\mathcal{P}$ under the right action of the group $U(n)$.

- The isotropy group is $U(n-m)$ so that

$$
\mathcal{C} \stackrel{\text { diff }}{\equiv} U(n) / U(n-m) \text {. }
$$

- The physical phase space $\mathcal{C} / U(m)$ is therefore diffeomorphic to

$$
\mathcal{P}_{\mathrm{ph}} \stackrel{\text { diff }}{\equiv} G_{m, n}(\mathbb{C})=U(n) /[U(n-m) \times U(m)] .
$$

Let us now turn to the complexification of the symplectic action of $U(m)$ in order to study the form that our general result (that the map (27) is a diffeomorphism) takes in this particular example. The complexification of $U(m)$ is

$$
U(m)_{\mathbb{C}}=G L(m, \mathbb{C}) .
$$

It is easy to verify that the action of $G L(m, \mathbb{C})$ on $\mathcal{P}=\mathbb{C}^{m n}$ by left multiplication

$$
L_{A^{\mathbb{C}} z}=A^{\mathbb{C}} z, \quad A^{\mathbb{C}} \in G L(m, \mathbb{C})
$$

is an extension of (69) in the sense of (12) and satisfies the conditions (15) and (16). Also, 0 is a regular value of the momentum map $\mu$ so that (27) is indeed a diffeomorphism. $\mathcal{C}^{\text {sat }}$ consists of all matrices $z \in \mathbb{C}^{m n}$ with $\operatorname{rank}(z)=m$. To prove this it is sufficient to notice that for any such matrix $z$ there is a matrix $A^{\mathbb{C}} \in G L(m, \mathbb{C})$ such that $A^{\mathbb{C}} z \in \mathcal{C}$ (e.g. by using the Graham-Schmidt orthogonalization procedure). Hence $\mathcal{C}^{\text {sat }}$ is dense in $\mathcal{P}=\mathbb{C}^{m n}$, which is in accordance with our conjecture. Furthermore, the "bad" points outside $\mathcal{C}^{\text {sat }}$ are points with symmetries (i.e. with a non-trivial isotropy subgroup of $U(m)$ ). 
The action of $G L(m, \mathbb{C})$ on $\mathcal{C}^{\text {sat }}$ is free by construction, and it is clear why the orbit space is diffeomorphic to the complex Grassmann manifold $G_{m, n}(\mathbb{C})$ : the orbits are just the sets of all bases of a given $m$-dimensional subspace of $\mathbb{C}^{n}$,

$$
\mathcal{P}_{\mathrm{ph}}=G_{m, n}(\mathbb{C})=\mathcal{C} / U(m)=\mathcal{C}^{\mathrm{sat}} / G L(m, \mathbb{C})
$$

\section{Application to Yang-Mills theory}

\subsection{The hamiltonian formulation}

In this section we review some geometric properties of the infinite-dimensional phase space of the Yang-Mills theory, which allow us to construct its complexification along the lines proposed in Section 3. We emphasize the geometric viewpoint of the theory, and refer the interested reader to Refs. [2,5,21] for the analytic details.

Let $\Sigma$ be a compact, oriented manifold of dimension three, $P=P(\Sigma, K)$ a principal fibre bundle over $\Sigma$, with structure group $K$, a compact semisimple Lie group, and associated Lie algebra $\mathcal{K}$. The Killing form in $\mathcal{K}$ will be used to identify $\mathcal{K}$ with its dual $\mathcal{K}^{*}$.

The big configuration space of Yang-Mills theory is the affine space $\mathcal{A}$ of $\mathcal{K}$-valued connections on $P$. For simplicity, we assume $P$ to be trivial, so that we can identify $\mathcal{A}$ with the affine space of $\mathcal{K}$-valued one-forms (gauge potentials) on $\Sigma$. (Here and in the following all function spaces are assumed to belong to the appropriate Sobolev classes, see [21] for a discussion.) Then $\mathcal{A}$ is an affine space modelled on the vector space $\bar{\Lambda}^{1}(\Sigma ; \mathcal{K})$ of $\mathcal{K}$-valued one-forms of adjoint type on $\Sigma$, and its tangent bundle can be identified with

$$
T \mathcal{A} \cong \mathcal{A} \times \bar{\Lambda}^{1}(\Sigma ; \mathcal{K})
$$

The corresponding Yang-Mills phase space will be identified with the $\left(L^{2}-\right)$ cotangent bundle

$$
T^{*} \mathcal{A} \cong \mathcal{A} \times \mathcal{X}_{d}(\Sigma ; \mathcal{K})
$$

where $\mathcal{X}_{d}(\Sigma ; \mathcal{K})$ denotes the space of $\mathcal{K}$-valued vector densities (or non-abelian electric fields) on $\Sigma$. At any point $A \in \mathcal{A}$, the dual pairing between $T_{A}^{*} \mathcal{A} \cong \mathcal{X}_{d}(\Sigma ; \mathcal{K})$ and $T_{A} \mathcal{A} \cong \bar{\Lambda}^{1}(\Sigma ; \mathcal{K})$ is given by

$$
(\alpha, \tilde{E})=\int_{\Sigma} \alpha: \tilde{E},
$$

where $\alpha \in T_{A} \mathcal{A}, \tilde{E} \in T_{A}^{*} \mathcal{A}$, and ":" denotes the complete contraction of internal and spatial indices (internal indices are contracted with the Killing form on $\mathcal{K}$ ).

The gauge group $G$ of Yang-Mills theory is the group of $K$-valued functions $g: \Sigma \rightarrow K$, on $\Sigma$, and its Lie algebra $\mathcal{G}$ is given by the Lie algebra of $\mathcal{K}$-valued functions $\xi: \Sigma \rightarrow \mathcal{K}$, on $\Sigma$. The dual of $\mathcal{G} \cong \Lambda^{0}(\Sigma ; \mathcal{K})$ is the space $\mathcal{G}^{*} \cong \Lambda_{d}^{0}(\Sigma ; \mathcal{K})$ of $\mathcal{K}$-valued scalar densities 
on $\Sigma$. The dual pairing between an algebra element $\xi \in \mathcal{G}$ and a scalar density $\tilde{\eta} \in \mathcal{G}^{*}$ is given by

$$
\prec \xi, \tilde{\eta} \succ=\int_{\Sigma} \xi: \tilde{\eta} .
$$

The group $G$ acts on the configuration space $\mathcal{A}$ according to the affine map

$$
(g, A) \mapsto \varphi_{g}(A)=g^{-1} A g+g^{-1} \mathrm{~d} g,
$$

whose canonical lift to the phase space $T^{*} \mathcal{A}$ yields the well-known Yang-Mills transformation law

$$
(g,(A, \tilde{E})) \mapsto \widetilde{\varphi}_{g}(A, \tilde{E})=\left(g^{-1} A g+g^{-1} \mathrm{~d} g, g^{-1} \tilde{E} g\right)
$$

The action (90) is symplectic with respect to the canonical (constant) symplectic form $\Omega$ on $T^{*} \mathcal{A}$,

$$
\Omega_{(A, \tilde{E})}\left(\left(\delta A_{1}, \delta \tilde{E}_{1}\right),\left(\delta A_{2}, \delta \tilde{E}_{2}\right)\right)=\left(\delta A_{1}, \delta \tilde{E}_{2}\right)-\left(\delta A_{2}, \delta \tilde{E}_{1}\right)
$$

for tangent vectors $\left(\delta A_{i}, \delta \tilde{E}_{i}\right) \in T_{(A, \tilde{E})}\left(T^{*} \mathcal{A}\right)$, where $(\cdot, \cdot)$ denotes the duality (87). Note that in this last equation we have used the identification

$$
T_{(A, \tilde{E})}\left(T^{*} \mathcal{A}\right) \cong \bar{\Lambda}^{1}(\Sigma ; \mathcal{K}) \times \mathcal{X}_{d}(\Sigma ; \mathcal{K}) .
$$

The infinitesimal generator of the configuration space action (89) associated to the algebra element $\xi \in \mathcal{G}$ is the vector field $\xi^{\mathcal{A}} \in \mathcal{X} \mathcal{A}$, given by

$$
\xi^{\mathcal{A}}(A)=\left(A, D_{A} \xi\right)=\left(D_{A} \xi\right) \frac{\delta}{\delta A},
$$

where $D_{A} \cdot=d \cdot+[A \wedge \cdot]$ is the covariant derivative defined by the connection $A$.

Analogous to the finite-dimensional case (cf. Section 2), the phase space action (90) admits an equivariant momentum map $\mu: T^{*} \mathcal{A} \rightarrow \mathcal{G}^{*} \cong \Lambda_{d}^{0}(\Sigma ; \mathcal{K})$, defined as

$$
\mu(A, \tilde{E}) \bullet \xi=\mu^{\xi}(A, \tilde{E})=\left(\xi^{\mathcal{A}}(A), \tilde{E}\right)=\left(D_{A} \xi, \tilde{E}\right)=\prec \xi, \delta_{A} \tilde{E} \succ,
$$

where $\delta_{A}$ denotes the (formal) adjoint of $D_{A}$, and $(\cdot, \cdot), \prec \cdot, \succ$ are the dualities (87) and (88) respectively. It follows that $\mu(A, \tilde{E})=\delta_{A} \tilde{E} \in \mathcal{G}^{*}$, and hence the Gauss constraint set $\mathcal{C}=\mu^{-1}(0)$ is given by all phase space points satisfying the Gauss law constraint

$$
\delta_{A} \tilde{E}=0 .
$$

The infinitesimal generator of the phase space action (90) associated to the algebra element $\xi \in \mathcal{G}$ is the vector field $\xi^{T^{*} \mathcal{A}} \in \mathcal{X}\left(\mathcal{T}^{*} \mathcal{A}\right)$, given by

$$
\xi^{T^{*} \mathcal{A}}(A, \tilde{E})=\left((A, \tilde{E}),\left(D_{A} \xi,[\tilde{E}, \xi]\right)\right)=\left(D_{A} \xi\right) \frac{\delta}{\delta A}+[\tilde{E}, \xi] \frac{\delta}{\delta \tilde{E}} .
$$

We now fix a Riemannian metric $h$ on the manifold $\Sigma$ and define an $h$-dependent, almostcomplex structure $\mathbf{J}$ on $T^{*} \mathcal{A}$ by its action on tangent vectors

$$
\mathbf{J}_{(A, \tilde{E})}:(\delta A, \delta \tilde{E}) \mapsto\left(-\delta E^{\mathfrak{b}}, \widetilde{\delta A^{\sharp}}\right),
$$


using the identification (92), the notation $\delta E^{b}$ for the $\mathcal{K}$-valued 1 -form $h$-equivalent to $\delta \tilde{E}$, and the notation $\widetilde{\delta A}$ for the $\mathcal{K}$-valued vector density $h$-equivalent to $\delta A$. In complete analogy with the finite-dimensional case of Eq. (16), this allows us to define a (weak) Riemannian metric $\gamma$ on the cotangent bundle $T^{*} \mathcal{A}$ via

$$
\begin{aligned}
\gamma\left(\left(\delta A_{1}, \delta \tilde{E}_{1}\right),\left(\delta A_{2}, \delta \tilde{E}_{2}\right)\right)_{(A . \tilde{E})} & :=\Omega_{(A, \tilde{E})}\left(\left(\delta A_{1}, \delta \tilde{E}_{1}\right), \mathbf{J}\left(\delta A_{2}, \delta \tilde{E}_{2}\right)\right) \\
& =\Omega_{(A, \tilde{E})}\left(\left(\delta A_{1}, \delta \tilde{E}_{1}\right),\left(-\delta E_{2}^{\mathrm{b}}, \widetilde{\delta A_{2}^{\sharp}}\right)\right) \\
& =\left(\delta A_{1}, \widetilde{\delta A_{2}}\right)+\left(\delta E_{2}^{\mathrm{b}}, \delta \tilde{E}_{1}\right) .
\end{aligned}
$$

Note that the almost-complex structure $\mathbf{J}$, and therefore also the Riemannian metric $\gamma$ are $G$-invariant. Since the actions (89) and (90) are affine, their differentials are the respective linear parts, and hence

$$
\begin{aligned}
\mathbf{J} \circ \mathrm{d} \widetilde{\varphi}_{g}(\delta A, \delta \tilde{E}) & =\mathbf{J}\left(g^{-1} \delta A g, g^{-1} \delta \tilde{E} g\right) \\
& =\left(g^{-1} \delta E^{b} g, g^{-1} \tilde{\delta A^{\ddagger}} g\right) \\
& =\mathrm{d} \varphi_{g} \circ \mathbf{J}(\delta A, \delta \tilde{E}),
\end{aligned}
$$

proving the $G$-invariance. The situation is formally the same as in Section 3, i.e. we have a $G$-invariant almost-Kähler structure $(\mathbf{J}, \gamma)$ obeying the fundamental relation

$$
\gamma(\cdot, \cdot)=\Omega(\cdot, \mathbf{J} \cdot)
$$

Considering as before the tangent map $\mathrm{d} \mu$ and its $\left(L^{2}\right.$-)adjoint $\nabla \mu=\mathrm{d} \mu^{*}$, using elliptic theory [2,5] we once more deduce the (gauge-invariant) Moncrief decomposition (25), this time of the tangent space $T_{p}\left(T^{*} \mathcal{A}\right)$, at any point $p=\left(A_{0}, \tilde{E}_{0}\right)$ of the Gauss constraint set $\mathcal{C}$.

In order that $\mathcal{C}$ be a manifold, we must exclude from it points $(A, \tilde{E})$ which possess one or more so-called infinitesimal symmetries, i.e. covariantly constant, non-zero functions $\xi \in \mathcal{G} \cong \Lambda^{0}(\Sigma ; \mathcal{K})$ that commute with the electric field $\tilde{E}$,

$$
D_{A} \xi=0 \text { and }[\tilde{E}, \xi]=0 .
$$

In a point $(A, \tilde{E})$ with infinitesimal gauge symmetries, the Yang-Mills field variables can be reduced to take their values in the Lie algebra of a smaller group $H \subset K$. Note that the kernel $\operatorname{Ker} \nabla \mu_{\left(A_{0}, \tilde{E}_{0}\right)}$ coincides with the set of infinitesimal symmetries of the configuration $\left(A_{0}, \tilde{E}_{0}\right)$. One can show that $\operatorname{Ker} \nabla \mu_{\left(A_{0}, \tilde{E}_{o}\right)}=\{0\}$ iff $\mathrm{d} \mu_{\left(A_{0}, \tilde{E}_{0}\right)}$ is surjective which, by the implicit function theorem, implies the following result [5].

Theorem 4. If $\left(A_{0}, \tilde{E}_{0}\right)$ has no infinitesimal symmetries, then the Gauss constraint set $\mathcal{C}=\mu^{-1}(0)$ is a manifold near $\left(A_{0}, \tilde{E}_{0}\right)$, with tangent space given by $\operatorname{Ker} \mathrm{d} \mu_{\left(A_{0}, \tilde{E}_{0}\right)}$.

We therefore exclude all points with symmetries from the Gauss constraint surface, and continue to denote the resulting smooth manifold by $\mathcal{C}$. In every point $\left(A_{0}, \tilde{E}_{0}\right)$ of this manifold, the operator $\nabla \mu_{\left(A_{0}, E_{0}\right)}$ has a trivial kernel and the "Laplacian" operator $\Delta=\mathrm{d} \mu_{\left(A_{0}, \tilde{E}_{0}\right)} \circ \nabla \mu_{\left(A_{0}, \tilde{E}_{0}\right)}$ is an isomorphism from $\mathcal{G} \cong \Lambda^{0}(\Sigma ; \mathcal{K})$ to $\mathcal{G}^{*} \cong \Lambda_{d}^{0}(\Sigma ; \mathcal{K})$. 
Marsden-Weinstein reduction leads to the reduced, physical phase space $\mathcal{P}_{\mathrm{ph}}=\mathcal{C} / G$, which is symplectomorphic to the cotangent bundle $T^{*} \mathcal{M}_{\mathrm{ph}}$ of the moduli space $\mathcal{M}_{\mathrm{ph}}=\mathcal{A}^{\prime} / G$ of equivalence classes of non-symmetric connections $\mathcal{A}^{\prime}$. Hence all aspects of the finitedimensional kinematic discussion of Section 2 are realized in the field-theoretic example of the Yang-Mills phase space.

\subsection{Complexification}

Let us denote by $\mathcal{A}^{\mathbb{C}}$ the complex affine space of $\mathcal{K}_{\mathbb{C}}$-valued connection one-forms on $\Sigma$ ( $\mathcal{K}_{\mathbb{C}}$ is the Lie algebra of the universal complexification $K_{\mathbb{C}}$ of $K$ introduced in Section 3 ). $\mathcal{A}^{\mathrm{C}}$ is the complexification of the real affine space $\mathcal{A}$, and is modelled on the vector space $\bar{\Lambda}^{1}\left(\Sigma ; \mathcal{K}_{\mathbb{C}}\right)$. The choice of a Riemannian metric $h$ on $\Sigma$ provides us with a one-to-one map $\mathcal{A}^{\mathbb{C}} \rightarrow T^{*} \mathcal{A}$, defined by

$$
A+\mathrm{i} E \mapsto\left(A, \tilde{E}^{\sharp}\right),
$$

where $\tilde{E}^{\sharp}$ denotes the $\mathcal{K}$-valued vector density $h$-equivalent to the $\mathcal{K}$-valued one-form $E .{ }^{3}$ This is well defined, since $A+\mathrm{i} E$ is a $K_{\mathbb{C}}$-valued one-form of $A_{K_{\mathbb{C}}}$-type, and in particular of $\operatorname{Ad}_{K}$-type. This implies that (restricting to the subgroup $K \subset K_{\mathbb{C}}$ ) $A$ is $K$-pseudotensorial and $\tilde{E}^{\sharp}$ of $\mathrm{Ad}_{K}$-type.

Following the construction given in Section 3, we now "complexify" the infinitesimal $\mathcal{G}$-action on $T^{*} \mathcal{A}$,

$$
\xi \mapsto \xi^{T^{*} \mathcal{A}}(A, \tilde{E})=\left(D_{A} \xi\right) \frac{\delta}{\delta A}+[\tilde{E}, \xi] \frac{\delta}{\delta \tilde{E}},
$$

by representing the imaginary generators according to

$$
\mathrm{i} \xi \mapsto \mathbf{J} \xi^{T^{*} \mathcal{A}} .
$$

Let us verify that the infinitesimal $G_{\mathbb{C}}$-gauge action on the complex Yang-Mills configuration space $\mathcal{A}^{\mathbb{C}}$ is compatible, under the map (102), with the infinitesimal "complexified" action on $T^{*} \mathcal{A}$, given by (103) and (104) (i.e. that Condition 1 of Section 3 holds). For the former, one has

$$
\begin{aligned}
(\xi+\mathrm{i} \eta) \cdot(A+\mathrm{i} E) & =D_{A+\mathrm{i} E}(\xi+\mathrm{i} \eta) \\
& =\mathrm{d}(\xi+\mathrm{i} \eta)+[(A+\mathrm{i} E),(\xi+\mathrm{i} \eta)] \\
& =D_{A} \xi-[E, \eta]+\mathrm{i}\left(D_{A} \eta+[E, \xi]\right),
\end{aligned}
$$

while for the latter one derives

\footnotetext{
${ }^{3}$ Since $A$ and $E$ have different physical dimension, we should be writing $A+\mathrm{i} L E$ instead of $A+\mathrm{i} E$ in (102), where $L$ is a fixed constant with the dimension of length. For simplicity we have set this constant equal to one.
} 


$$
\begin{aligned}
(\xi+\mathrm{i} \eta) \cdot\left(A, \tilde{E}^{\sharp}\right) & =\xi \cdot\left(A, \tilde{E}^{\sharp}\right)+\mathbf{J}\left(\eta \cdot\left(A, \tilde{E}^{\sharp}\right)\right) \\
& =\left(D_{A} \xi,\left[\tilde{E}^{\sharp}, \xi\right]\right)+\mathbf{J}\left(D_{A} \eta,\left[\tilde{E}^{\sharp}, \eta\right]\right) \\
& =\left(D_{A} \xi-[E, \eta],\left[\tilde{E}^{\sharp}, \xi\right]+\widetilde{D_{A}} \eta^{\sharp}\right) .
\end{aligned}
$$

Clearly, the right-hand side of (105) is mapped into the right-hand side of (106) under the map (102). We have therefore verified the claim made in Section 3 above, that the kinematic, complex Yang-Mills theory with structure group $K_{\mathbb{C}}$ satisfies Definition 1 and Condition 1 with respect to its real counterpart with structure group $K$.

The validity of the Moncrief decomposition (25) and the inverse mapping theorem (in the appropriate weighted Sobolev spaces [21]), and the fact that (98) is a weak Riemannian metric in $T^{*} \mathcal{A}$ imply that Propositions 1 and 2 of Section 3 are valid in the present case and therefore the following theorem (the analogue of Theorem 3 for the infinite-dimensional case of a Yang-Mills theory) holds.

Theorem 5. Consider a Yang-Mills theory with compact structure group $K$ corresponding to the trivial bundle $P(\Sigma, K)$ over the three-dimensional oriented and compact manifold $\Sigma$. Then the map (27) is a diffeomorphism between the physical phase space $\mathcal{C} / \tilde{L}_{G}$ of the Yang-Mills theory with structure group $K$ and an open submanifold $\mathcal{C}^{\mathrm{sat}} / L_{G_{\mathbb{C}}}$ of the physical configuration space of the Yang-Mills theory with structure group $K_{\mathbb{C}}$ (assuming that points with symmetries have been excluded from $\mathcal{C}$ ).

\subsection{Holomorphic Wilson loops}

With these ingredients in hand, we can now form the saturation $\mathcal{C}^{\text {sat }}$ of the Gauss constraint manifold $\mathcal{C}$ according to formula (26). If we can show that any $G_{\mathbb{C}}$-orbit through a point $A^{\mathbb{C}} \in \mathcal{A}^{\mathbb{C}}$ cuts the constraint surface $\mathcal{C}$, we have $\mathcal{C}^{\text {sat }} \cong \mathcal{A}^{\mathbb{C}}$, and thus an equivalence of the quotient spaces

$$
\mathcal{C} / G \cong \mathcal{A}^{\mathbb{C}} / G_{\mathbb{C}}
$$

Note that, due to the geometric structures available on $\mathcal{A}^{\mathbb{C}}$, we do never have the problem of uniqueness of the "gauge choice" $\delta_{A} \tilde{E}=0$, the Gauss law constraint of Eq. (95), i.e. a given $G_{\mathbb{C}}$-orbit can never cut the surface $\mathcal{C}$ more than once. For the attainability of this "gauge choice", one derives the following conditions. Starting from an arbitrary point $A^{\mathbb{C}}=A+\mathrm{i} E \in \mathcal{A}^{\mathbb{C}}$, one looks for a complex gauge transformation $g_{\mathbb{C}}=g \cdot \exp i \omega \in$ $G_{\mathbb{C}}$, such that the configuration $\left(A^{\prime}, \tilde{E}^{\prime}\right)$, where $\varphi_{g_{\mathbb{C}}}(A+\mathrm{i} E)=A^{\prime}+\mathrm{i} E^{\prime}$, lies in $\mathcal{C}$. Taking w.l.o.g. $g_{\mathbb{C}}$ to be purely imaginary, $g_{\mathbb{C}}=\mathrm{e}^{\mathrm{i} \omega}$, one derives the following non-linear equations for $\omega$ :

$$
\begin{aligned}
& A^{\prime}=\sum_{n=0}^{\infty} \frac{(-1)^{n}}{(2 n) !}(\operatorname{Ad} \omega)^{2 n} A-\sum_{n=0}^{\infty} \frac{(-1)^{n+1}}{(2 n+1) !}(\operatorname{Ad} \omega)^{2 n+1}(E+\mathrm{d} \omega) \\
& E^{\prime}=\sum_{n=0}^{\infty} \frac{(-1)^{n+1}}{(2 n+1) !}(\operatorname{Ad} \omega)^{2 n+1} A+\sum_{n=0}^{\infty} \frac{(-1)^{n}}{(2 n) !}(\operatorname{Ad} \omega)^{2 n}(E+\mathrm{d} \omega)
\end{aligned}
$$


using the notation

$$
(\operatorname{Ad} \omega)^{n} X:=[\omega,[\omega, \ldots,[\omega,[\omega, X]] \ldots]], \quad(\operatorname{Ad} \omega)^{0} X:=X
$$

Comparing with the finite-dimensional $S O(n)$-gauge model, one may not expect the Eqs. (108) to possess solutions for arbitrary $(A, E)$, but still these relations may be useful in determining whether $\mathcal{C}^{\text {sat }}$ is dense in $\mathcal{A}^{\mathbb{C}}$, as we are conjecturing.

Recall there is a natural set of gauge-invariant variables on any space $\mathcal{A}$ of connections, given by the so-called Wilson loops

$$
T_{\gamma}(A):=\operatorname{Tr} P \exp \oint_{\gamma} A,
$$

where $\gamma$ is a closed curve in $\Sigma$, and $P$ denotes path-ordering along $\gamma$. The expression $P \exp \oint_{\gamma} A$ is also known as the holonomy of the connection $A$ along the loop $\gamma$.

It is well known that for compact structure group $K$, the knowledge of the values of all Wilson loops $T_{\gamma}$ is equivalent to the knowledge of the gauge connection $A$ up to gauge transformations [11], and the variables $\left\{T_{\gamma}\right\}$ form an overcomplete set of coordinates on the physical configuration space $\mathcal{A} / G$. For non-compact $K$, the Wilson loops may not be completely separating, i.e. there may be sets of connection configurations from $\mathcal{A} / G$ that are mapped into the same $T_{\gamma}$-configuration. However, for the case $K_{\mathbb{C}}=S L(2, \mathbb{C})=S U(2)_{\mathbb{C}}$, which is the one relevant for the Ashtekar formulation of $3+1$ gravity, the points in $\mathcal{A}^{\mathbb{C}} / G_{\mathbb{C}}$ which are not separated by the Wilson loop variables form a set of measure zero [12], and moreover the Wilson loops separate all points which can be separated in the non-Hausdorff space $\mathcal{A}^{\mathbb{C}} / G_{\mathbb{C}}[7]$.

Let us now consider the traced holonomies (110) as functions on the space $\mathcal{A}^{\mathbb{C}} / G_{\mathbb{C}}$ of complex gauge connections modulo gauge transformations introduced earlier (see [25]). As mentioned above these variables have been proven in [12,7] to form a set of good generalized coordinates on the physical configuration space of the Yang-Mills theory with complex structure group $K_{\mathbb{C}}=S L(2, \mathbb{C})$. Our Propositions 1 and 2 and Theorem 5 provide a set of necessary conditions for the variables $(110)$ to be good generalized coordinates on the physical phase space of the Yang-Mills theory with structure group $S U$ (2). (A proof that $\mathcal{C}^{\text {sat }}$ is dense would provide a sufficient condition.) This is also relevant to general relativity written in terms of Ashtekar variables, since there $\mathcal{A}^{\mathbb{C}}$ is the (big) phase space rather than the (big) configuration space of the theory. Although the symplectic structure of general relativity is very different from that of $S U(2)$ Yang-Mills theory, one may expect that an analogue of our result is valid also in the case of gravity. This would indicate that (110) are good generalized coordinates on the phase space of general relativity in the Ashtekar formulation (complementing the results of $[12,7]$ ). A proof of this assertion would however involve a proof of Theorem 5 and of the conjecture that $\mathcal{C}^{\text {sat }}$ is dense also for the gravity case, which is beyond the scope of the present paper.

On the other hand, our result points to an alternative to the usual loop space formulations of Yang-Mills theory, where the Wilson loops (110) are used as variables on the physical 
configuration space, and where generalized Wilson loops have to be introduced in a rather asymmetric way to bring in the dependence on the canonically conjugate momenta $\tilde{E}$ [10].

A next important step in our construction is the search for natural algebraic structures on the set of holomorphic and anti-holomorphic Wilson loops, which could serve as a starting point for the quantization.

\section{Conclusions}

We have derived a number of conditions, under which it is possible to obtain an alternative description for the reduced phase space of a hamiltonian first-class constrained system. Instead of using the two-step Marsden-Weinstein reduction associated with the group $G$ of gauge transformations, one takes a single quotient with respect to an appropriate phase space action of the complexified group $G_{\mathbb{C}}$ (Theorems 3 and 5). A necessary condition for these two methods to lead to equivalent results is the existence of an appropriate extension of the $G$-action in the directions perpendicular to the constraint surfaces.

We conjecture that the conditions for equivalence we establish are actually sufficient, and hence that the saturation $\mathcal{C}^{\text {sat }}$ (all points in phase space that can be reached from the zero-momentum constraint surface $\mathcal{C}$ by a complex gauge transformation from $G_{\mathbb{C}}$ ) is dense in the phase space. This conjecture is corroborated by all the finite-dimensional examples investigated in Section 4 , but a general proof is still lacking. The examples with $G=S O(n)$ and $G=U(m)$ also demonstrate that no obstructions in principle occur when the $G$-action is non-free or the phase space not of the form of a cotangent bundle over the configuration space.

Due to the dual interpretation of the space underlying our construction (either as the phase space $\mathcal{P}$ of a (real) gauge system or as the configuration space $Q^{\mathbb{C}}$ of a (complex) gauge system), it allows for a variety of physical applications, already outlined in the introduction. The infinite-dimensional gauge theoretic case constituted the main motivation for this work, in view of an extension to the case of 3+1-dimensional Ashtekar relativity, which is momentarily under investigation. An application of our result to Chern-Simons theory for simple spatial topologies can be found in [18]. In any of these cases one has to show that a (hamiltonian or lagrangian) dynamics can be introduced into the framework in a consistent way. We leave the discussion of this and further applications to a future publication.

\section{Acknowledgements}

We are grateful to A. Ashtekar, R. Picken and A.R. Silva for useful discussions and to J. Rawnsley for a comment. JNT and JMM were partially supported by JNICT grant PESO/P/PRO/8/91 and GTAE, and RL partially by a DFG scholarship. 


\section{References}

[1] R. Abraham and J.E. Marsden, Foundations of Mechanics, 2nd Ed. (Benjamin/Cummings, Reading, 1978).

[2] J.M. Arms, The structure of the solution set for the Yang-Mills equations, Math. Proc. Camb. Phil. Soc. 90 (1981) 361-372.

[3] J.M. Arms, Symmetry and solution set singularities in Hamiltonian field theories, Acta Phys. Pol. B 17 (1986) 499-523.

[4] J.M. Arms, A. Fischer and J.E. Marsden, Une approche symplectique pour des théorèmes de décomposition en géométrie ou relativité générale, C. R. Acad. Sci. Paris 281 (1975) 517-520.

[5] J.M. Arms, J.E. Marsden and V. Moncrief, Symmetry and bifurcations of momentum mappings, Comm. Math. Phys. 78 (1981) 455-478.

[6] A. Ashtekar, Lectures on Non-Perturbative Canonical Gravity (World Scientific, Singapore, 1991).

[7] A. Ashtekar and J. Lewandowski, Completeness of Wilson loop functionals on the moduli space of $S L(2, \mathbb{C})$ and $S U(1,1)$-connections, Class. Quant. Grav. 10 (1993) L69-74.

[8] A. Ashtekar and J.D. Romano, Chern-Simons and Palatini actions and (2+1)-gravity, Phys. Lett. B 229 (1989) 56-60.

[9] C. Emmrich and H. Römer, Orbifolds as configuration spaces of systems with gauge symmetries, Comm. Math. Phys. 129 (1990) 69-94.

[10] R. Gambini and A. Trias, Gauge dynamics in the $C$-representation, Nucl. Phys. B 278 (1986) 436-448.

[11] R. Giles, Reconstruction of gauge potentials from Wilson loops, Phys. Rev. D 24 (1981) 2160-2168.

[12] J.N. Goldberg, J. Lewandowski and C. Stornaiolo, Degeneracy in loop variables, Comm. Math. Phys. 148 (1992) $377-402$.

[13] M.J. Gotay, Constraints, reduction and quantization, J. Math. Phys. 27 (1986) 2051-2066.

[14] V. Guillemin and S. Sternberg, Symplectic Techniques in Physics (Cambridge University Press, Cambridge, 1984).

[15] N.J. Hitchin, The self-duality equations on a Riemann surface, Proc. London Math. Soc. 55(3) (1987) 59-126.

[16] G. Hochschild, The Structure of Lie Groups (Holden-Day, San Francisco, 1965).

[17] F.C. Kirwan, Cohomology of Quotients in Symplectic and Algebraic Geometry, Mathematical Notes, Vol. 31 (Princeton University Press, Princeton, 1984).

[18] R. Loll, J.M. Mourão and J.N. Tavares, Symplectic reduction via complex group actions, in: Constraint Theory and Quantization Methods, ed. F. Colomo et al. (World Scientific, Singapore, 1994) pp. 291-304.

[19] J.E. Marsden and A. Weinstein, Reduction of symplectic manifolds with symmetry, Rep. Math. Phys. 5 (1974) $121-130$.

[20] V. Moncrief, Decompositions of gravitational perturbations, J. Math. Phys. 16 (1975) 1556-1560.

[21] V. Moncrief, Gribov degeneracies: Coulomb gauge conditions and initial value constraints, J. Math. Phys. 20 (1979) 579-585.

[22] R. Montgomery, The structure of reduced cotangent phase spaces for non-free group actions, Univ. of California, Berkeley, Math. preprint (1983).

[23] L. Ness (with an appendix by D. Mumford), A stratification of the null cone via the moment map, Amer. J. Math. 106 (1984) 1281-1329.

[24] L.V. Prokhorov, Hamiltonian path integrals, Sov. J. Part. Phys. 13 (1982) 456-482; L.V. Prokhorov and S.V. Shabanov, Phase space of mechanical systems with a gauge group. Sov. Phys. Uspekhi 34 (1991) $108-140$.

[25] C. Rovelli and L. Smolin, Loop space representation of quantum general relativity, Nucl. Phys. B 331 (1990) 80-152.

[26] K. Sjamaar, Holomorphic slices, symplectic reduction and multiplicities of representations, MIT preprint (1993).

[27] E. Witten, (2+1) dimensional gravity as an exactly soluble system, Nucl. Phys. B 311 (1988) 46-78.

[28] E. Witten, Quantization of Chern-Simons gauge theory with complex gauge group, Comm. Math. Phys. 137 (1991) 29-66. 